\title{
THE COMPARATIVE SENSITIVENESS OF SOME COMMON DETECTORS OF ELECTRICAL OSCILLATIONS
}

\author{
By Louis W. Austin.
}

Although probably many comparisons of the sensitiveness of detectors have been made in the laboratories of the wireless companies, very little quantitative information on this subject has been made public. For this reason the experiments here described have been carried out. On account of the difficulties and the amount of time involved in the testing of detectors in wireless stations, methods have been developed during the last three years whereby these tests can be made in the laboratory more expeditiously and accurately than is possible in the regular stations. The difficulties usually encountered in laboratory tests of this kind are due mainly to the direct effect of the source of the oscillations on the receiving circuits and detector; but by using properly designed exciting circuits in connection with a buzzer as a source of oscillations these difficulties have been removed. As it is also possible in this way to produce oscillations of any required degree of damping as well as of any required wave length, all of the problems of wireless telegraphy can be successfully investigated in the laboratory except those which have to do with the properties of different types of antennæ and the passage of the waves from station to station. In the following paper some experimental comparisons of the sensitiveness of wireless detectors are described.

SYMBOLS

$D=$ Galvanometer deflection In general proportional to $A=$ Telephone audibility $\}$ energy in detector.

$I=$ Oscillatory current.

$c=$ Direct current. 
$R=$ Direct current resistance.

$R^{\prime}=$ High frequency resistance.

$L_{1} L_{2}$, etc. $=$ Inductances.

$C_{1} C_{2}$, etc. $=$ Tuning condensers.

$K=$ Large fixed condenser 0.04-0.I $\mathrm{mf}$.

$M_{34}=$ Mutual inductance between coils 3 and 4 .

$d_{12}=$ Distance between coils I and 2 .

$\delta_{1}=$ Logarithmic decrement in circuit I.

$\lambda=$ Wave length.

\section{METHOD OF EXPERIMENT}

The exciting circuit ${ }^{1}$ in all the experiments is shown in Fig. I. Here $B$ is a buzzer run by two storage cells with considerable resistance in series. The oscillatory circuit consists of a variable air condenser $C_{1}$ having a maximum capacity of 0.005 microfarads, and for moderate wave lengths an inductance $L_{1}$ of 0.062 millihenries. These are connected directly across the contact of the buzzer.

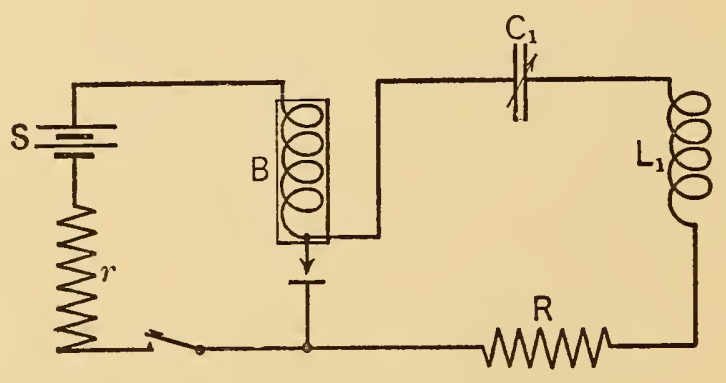

Fig. 1

The action of the circuit is as follows: When the buzzer contact is broken, the condenser $C_{1}$ is charged and this charge is retained until the contact is again closed, when the condenser discharges through the contact, producing oscillations in the circuit $L_{1} C_{1}$. As these oscillations take place in a closed metallic circuit, they are very feebly damped unless extra resistance is introduced at $R$.

All buzzers are not equally suited to producing oscillations by this method. In some, considerable adjustment of the contact is required before sharp tuning is obtained. The buzzer used in

${ }^{1}$ Other forms of buzzer circuit such as are used in several well-known types of wave meters were also tried, but with the buzzer used they gave less powerful oscillations than the one here shown. 
these experiments was of the 1905 United States Signal Corps type, and gave very satisfactory results with wave lengths varying between 350 and 2000 meters. With some care in adjustment the wave length could be pushed up beyond 3000 . For telephonic reception of signals it was usually found necessary to cut down the intensity of oscillations from the buzzer, and this was done by placing an incandescent lamp across the buzzer contact.

The chalcopyrite-zincite rectifier known as the perikon detector ${ }^{2}$ was used as a standard for the comparison of the various detectors. As its sensitiveness varies somewhat from time to time, it was standardized before each experiment in the following manner, the circuits being shown in Fig. 2. Here $I$ is the buzzer circuit, $I I$ a
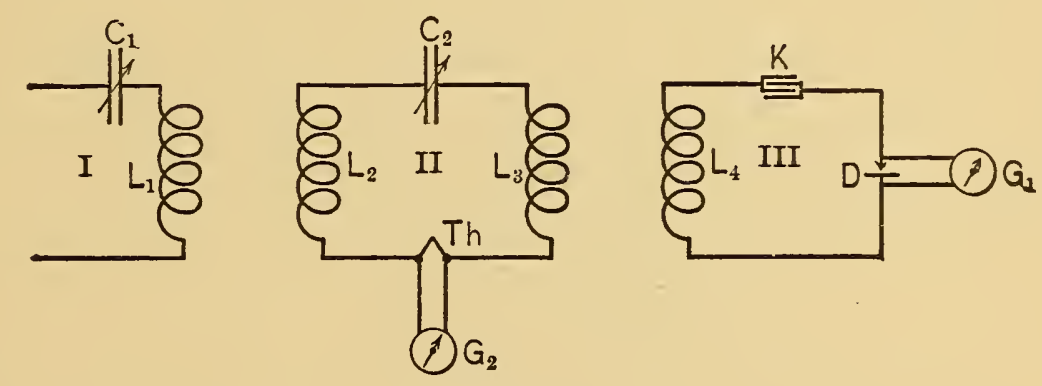

Fig. 2

circuit containing a thermoelement of known sensitiveness, and III the circuit consisting of the perikon $D$, its coupling inductance $L_{4}$, and a suitable stopping condenser $K$ of about 0.04 microfarad. With a given loose coupling between $L_{3}$ and $L_{4}$ the relation was noted between the reading of the galvanometer attached to the thermoelement and that attached to the perikon, ${ }^{3}$ the condition of wave length and damping being kept constant. This relation gives at once the relative sensitiveness of the perikon. It was found better to take account of the variations in sensitiveness observed rather than to readjust each time to the same sensitiveness.

Table I shows the admirable proportionality between the deflections of the perikon detector and the square of the oscillatory current as measured by the thermoelement.

\footnotetext{
${ }^{2}$ The perikon was a new one which had been little used and was of normal sensitiveness.

${ }^{3}$ The resistance in the perikon galvanometer circuit must be equal to that to be used in the experiment.
} 
TABLE I

Comparison of Tellurium-Constantan Thermoelement and Perikon Rectifier

(See Fig. 2)

\begin{tabular}{c|c|c}
\hline $\begin{array}{c}\text { Thermoelement, Circuit II } \\
\text { D }\end{array}$ & $\begin{array}{c}\text { Perikon, Circuit III } \\
\text { D }\end{array}$ & Ratio \\
\hline 2.3 & 25 & 10.9 \\
5.3 & 54 & 10.2 \\
9.7 & 100 & 10.3 \\
19.8 & 198 & 10.0 \\
31.2 & 330 & 10.6 \\
41.3 & 480 & 10.9 \\
51.7 & 540 & 10.4 \\
\hline
\end{tabular}

$$
\begin{array}{rlr}
L_{2} & =0.03 \mathrm{mh} \quad \lambda=900 \mathrm{~m} \\
L_{3} & =0.425 \quad \delta_{1}=0.16 \\
L_{4} & =0.420 \quad \delta_{\mathrm{II}}=0.13 \\
M_{34} & =0.025 \\
& \text { Circuit III } & \\
&
\end{array}
$$

Two general methods have been used for measuring the sensitiveness for telephonic reception of radiotelegraphic receivers: First, the shunted telephone method in which the relative loudness of response to the same signal in two different receivers can be compared, and, second, the variable coupling method by means of which the least audible signals for the different receivers can be compared.

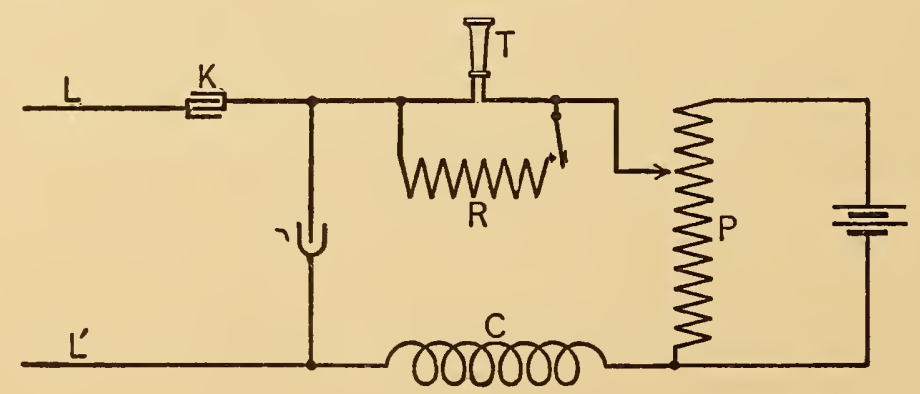

Fig. 3

A form of circuit for the shunted telephone method in the case of the electrolytic detector is shown in Fig. 3. Here $L$ and $L^{\prime}$ are wires leading to the oscillatory circuit, $K$ a stopping condenser of 0.04 microfarads capacity, $T$ the telephones, $R$ a vari- 
able resistance in shunt across the telephones, and $C$ a choke coil to prevent the oscillations from running around through $R$ instead of passing through the detector when the shunt $R$ is closed. Two 6o-ohm telephones form a suitable choke. Whatever choke coil is used it is necessary to test it by placing it across $L L^{\prime}$. If the' choke is perfect, no oscillations will pass through it, and when it is placed across $L L^{\prime}$ no change in the loudness of the signals will be observed.

The measurement of the intensity of signal is made as follows: After the receiving circuit and detector are adjusted to give maximum loudness in the telephone the shunt resistance $R$ is closed and the resistance regulated until the signal just becomes audible. The value of the current pulses $c$ in the telephone, which are proportional to the energy of the incoming waves in the detector, is expressed by the following well-known formula, where $r$ is the value of the shunt, and $t$ is the resistance of the telephones, and $c^{\prime}$ the least current audible in the telephones:

$$
c=\frac{r+t}{r} c^{\prime}
$$

The audibility, $A=\frac{c}{c^{\prime}}=\frac{r+t}{r}$.

The signal is often expressed in terms of $A$ as being so many times audibility. With care a series of measurements of intensity may be made to agree among themselves to within about ro per cent.

In the variable coupling method for comparing the least audible signals in two detectors, the deflections of the galvanometer connected to the perikon are observed for a number of different degrees of coupling between the inductances $L_{1}$ and $L_{2}$. (See Fig. 2.) From these a curve is plotted which gives the variation of the received energy as determined by the distance between the coils. The coupling, for which the signals just become audible in the telephones of the detectors being compared, is then observed and the relative energy taken from the curve.

Fig. 2 shows the general plan of the circuits used in the comparison of the detectors. $I$ is the exciting circuit adjusted to send 
out wave trains having a wave length group frequency and damping similar to those occurring in wireless practice. II is a circuit whose constants were made to correspond to a receiving antenna of moderate size. III is an untuned circuit containing a detector connected to a galvanometer or to a pair of head telephones, as the circumstances of the work required. The electrolytic, perikon, and audion were used in circuit $I I I$, each being so coupled to circuit $I I$ as to give maximum effect. The magnetic detector was placed in circuit $I I$, as it is generally used in the antenna. The Fleming vacuum valve detector was coupled to $I I$ by its own fixed coupling coils.

TABLE II

Electrolytic and Perikon Detectors, Shunted Telephone Method

\begin{tabular}{|c|c|c|c|}
\hline & & \multicolumn{2}{|c|}{ Relative sensitiveness } \\
\hline & & Strong signals & Weak signals \\
\hline$\frac{\text { Electrolytic }}{\text { Perikon }}$ & 4 & 2.5 & 2.4 \\
\hline$\frac{\text { Electrolytic }}{\text { Perikon }(0.2 \text { volt })}$ & 4 & 1.1 & 1.3 \\
\hline$\frac{\text { Electrolytic }}{\text { Perikon }}$ & 5 & 2.3 & 2.5 \\
\hline$\frac{\text { Electrolytic }}{\text { Perikon }(0.2 \text { volt })}$ & 5 & 1.3 & 1.3 \\
\hline
\end{tabular}

Telephone resistance $2100 \mathrm{ohms}$

\begin{tabular}{|c|c|c|}
\hline$\frac{\text { Electrolytic }}{\text { Perikon }}$ & 5 & 2.7 \\
\hline$\frac{\text { Electrolytic }}{\text { Perikon }(0.2 \text { volt })}$ & 5 & 1.1 \\
\hline
\end{tabular}

Telephone resistance $813 \mathrm{ohms}$

Inductances, decrements, and wave length as in Table I. $M_{34}$ adjusted for maximum sensitiveness. 
THE ELECTROLYTIC DETECTOR

Comparison was made of the sensitiveness of the electrolytic detector and the perikon, using a wave length of approximately 900 meters and a decrement of the waves given out by the exciter circuit, amounting to o.I6. The values of the inductances and capacities of the circuits were so chosen that they simulated the conditions of commercial work.

In Table II are given the ratio of sensitiveness for both strong and weak signals. The perikon was used without external EMF and also with about 0.2 of a volt applied in the direction of the rectification. Comparison was made by the shunted telephone method, the same telephones being used for both detectors. In one set of experiments the telephones used were of 2100 ohms and in another of $813 \mathrm{ohms}$. The relative sensitiveness of the two detectors was little affected by this change, showing that their effective resistances must be about the same. It is seen that without external EMF the perikon is less than half as sensitive as the electrolytic, but that when the 0.2 volt is applied the difference is very small. The electrolytic used in the experiment was of the free-wire type and two positive Wollaston wires were used during the test, one 0.003 and one $0.0012 \mathrm{~mm}$ in diameter. This was done because it has been frequently stated that the finer wire was more sensitive to weak signals. No marked difference between the two, however, could be detected. This shows that it is only necessary for sensitiveness, that the capacity reactance for the given frequency should be large, and that beyond a certain limiting diameter there is no object in reducing the size of the positive wire.

Table III shows the relation between galvanometer deflections with the thermoelement in circuit $I I$ and the intensity of signals in the electrolytic in circuit III as measured by the shunted telephone method. ${ }^{6}$ This shows that the responses of the electrolytic as indicated by the telephone are proportional to the square of the oscillatory current. ${ }^{7}$

${ }^{6} \mathrm{~A}$ comparison was also made of the sensitiveness of the perikon without external EMF and the electrolytic at the Washington Navy-Yard wireless station using incoming signals from a distant station. The results agreed within a few per cent with those found in the laboratory.

${ }^{7}$ Very strong signals, i. e., more than about 300 times audibility, sometimes give irregular results. 


\section{TABLE III}

Electrolytic

Received Energy and Audibility of Signals

\begin{tabular}{c|c|c}
\hline $\begin{array}{c}\text { Thermoelement in Circuit II } \\
\text { D }\end{array}$ & $\begin{array}{c}\text { Electrolytic in Circuit III } \\
\text { A }\end{array}$ & Ratio \\
\hline 3.3 & 12 & 36 \\
5.6 & 22.4 & 40 \\
3.4 & 140 & 41 \\
6.3 & 250 & 40 \\
8.0 & 324 & 41 \\
17.0 & 645 & 38 \\
\hline
\end{tabular}

Inductances, decrements, and wave length as in Table I. $M_{34}=0.025$.

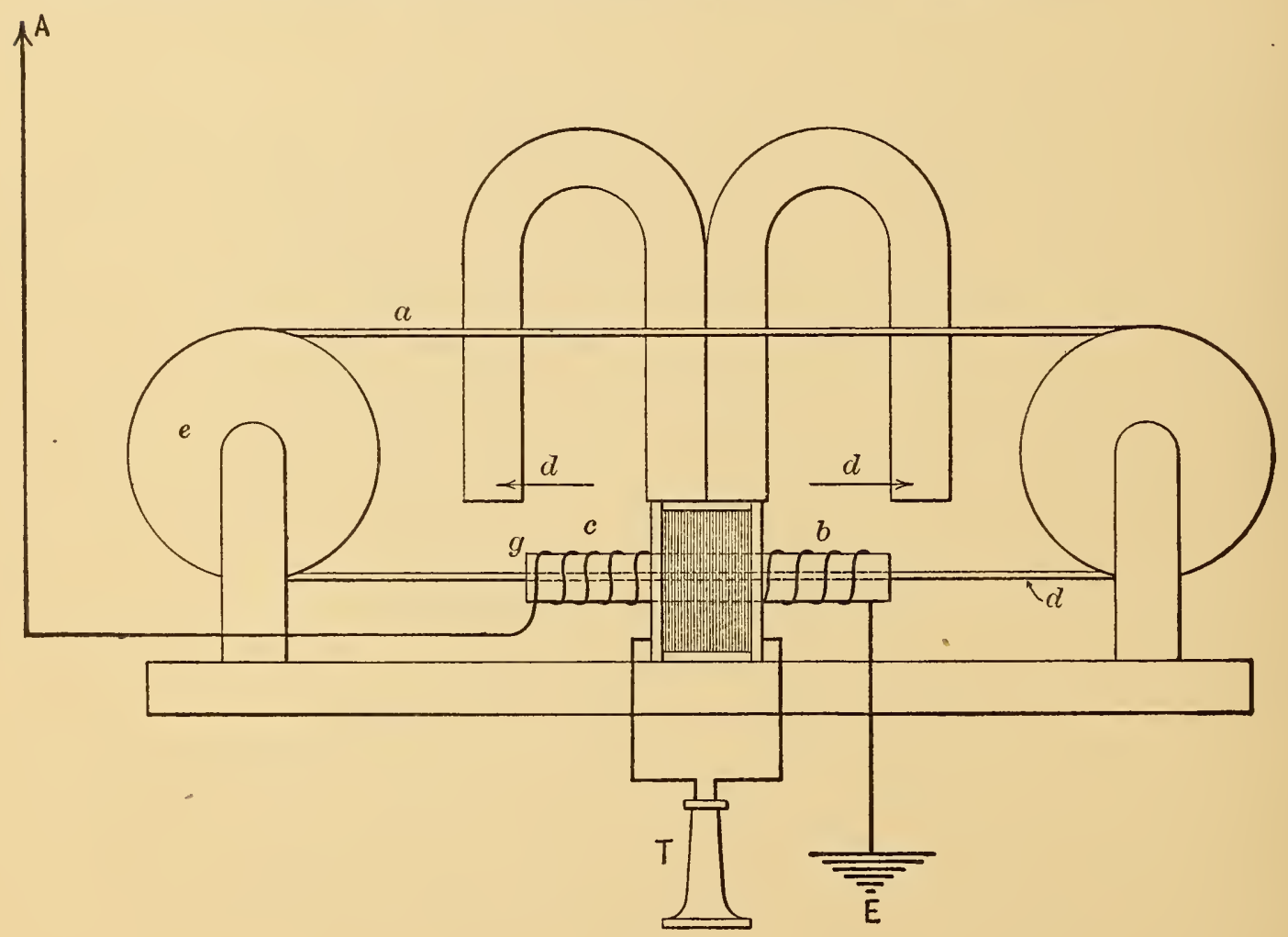

Fig. 4

THE MAGNETIC DETECTOR. (Fig. 4)

The circuits for the comparison of the magnetic and perikon detectors are shown in Fig. 2. The magnetic detector was connected directly in circuit $I I$ since it is usually used in the antenna. The magnetic detector used was of the ordinary. 
commercial type, the D. C. resistance of the oscillatory coil being $9.7 \mathrm{ohms}$ and the telephone coil $\mathrm{I} 2 \mathrm{O}$ ohms. The telephones furnished with the magnetic detector and used in the experiments had a resistance of $168 \mathrm{ohms}$. The telephones used with the perikon were of $\mathrm{I} 200 \mathrm{ohms}$ resistance and medium sensitiveness. For the comparison of sensitiveness the variable coupling method was used, the coupling $L_{1}$ and $L_{2}$ being changed until the signals became inaudible. When the readings on the magnetic were being taken the circuit containing the perikon was removed, then for the perikon readings the magnetic was cut out and the coupling $L_{3} L_{4}$ regulated for maximum strength of signals. The relative strength of signals for the different degrees of coupling between $L_{1}$ and $L_{2}$ was determined by means of the deflection of a galvanometer attached to the perikon. Such a series of readings is shown in Table IV.

\section{TABLE IV}

Relative Energy in Circuit II for Different Distances $d_{12}$ Between $L_{1}$ and $L_{2}$ (See Fig. 2)

\begin{tabular}{|c|c|}
\hline$d_{12}$ & $\underset{\mathrm{D}}{\text { Perikon }}$ \\
\hline $10 \mathrm{~cm}$ & 400 \\
\hline 12 & 245 \\
\hline 14 & 172 \\
\hline 16 & 115 \\
\hline 18 & 75 \\
\hline 20 & 54 \\
\hline 24 & 30 \\
\hline 28 & 14 \\
\hline 32 & 8 \\
\hline 38 & 3 \\
\hline
\end{tabular}

With waves from the buzzer circuit having a damping of 0.I 6 and a wave length of about 900 meters, it was found that the magnetic detector required 2.I times as much energy to produce an audible sound as the perikon used without external EMF. 
Further experiments showed that by putting a 0.05 microfarad condenser in series with the telephone of the magnetic, thus tuning it roughly to the rate of the buzzer, the loudness of signals could be increased so that there was very little difference between it and the perikon.

\section{TABLE V}

Magnetic and Perikon Detectors

Weak Signals

\begin{tabular}{c|c}
\hline$\lambda$ & Ratio of sensitiveness: $\frac{\text { Magnetic }}{\text { Perikon }}$ \\
\hline $350 \mathrm{~m}$ & 0.22 \\
900 & 0.9 \\
2000 & 1.0 \\
3000 & 1.5 \\
\hline
\end{tabular}

Comparisons were also made at a wave length of 350 meters and at 2000 and 3000 meters. At 3000 meters the magnetic with tuned telephone was approximately one and one-half times as sensitive as the perikon. At 2000 there was very little difference, while at 350 meters the perikon was nearly five times as sensitive as the magnetic.

In order to explain these differences in relative sensitiveness, the logarithmic decrement of the circuit containing the magnetic was determined for different frequencies. For this purpose the circuits were those shown in Fig. 2, the magnetic detector being in its place in circuit $I I$ and the perikon with its galvanometer in the untuned circuit $I I I$, very loosely coupled to $I I$, so that no appreciable energy was taken up by it. The buzzer exciter circuit was adjusted so as to give feebly damped oscillations having a decrement of about 0.02. The decrement of circuit II was determined from the well-known formula

$$
\delta_{I}+\delta_{I I}=\pi \frac{C_{m}-C}{C_{m}} \sqrt{\frac{I^{2}}{I_{m}^{2}-I^{2}}}
$$

where $C_{m}$ represents the reading of the condenser in circuit $I I$ for resonance and $C$ any other reading slightly removed from reso- 
nance. $I_{m}$ then represents the value of the oscillatory current for resonance and $I$ that for the setting of the condenser at $C$. This formula becomes much more convenient in practice if we choose $I^{2}$ so that it is just one-half $I_{m}{ }^{2}$, for then the expression under the radical reduces to unity. As the deflection of the galvanometer connected to the perikon is proportional to $I^{2}$, our expression becomes

$$
\delta_{I}+\delta_{I I}=\pi \frac{C_{m}-C}{C_{m}}
$$

where the change from $C_{m}$ to $C$, either an increase or a decrease, is just sufficient to reduce the deflection of the galvanometer to one-half. The mean of several determinations of $C_{m}-C$ gives as accurate values for the decrement as can be obtained from acurve plotted in the usual manner and calculated according to the complete formula. In damping experiments it is necessary that all

TABLE VI

Effective Resistance of Magnetic Detector at Different Wave Lengths

\begin{tabular}{c|c}
\hline$\lambda$ & \multicolumn{1}{c}{$\mathrm{R}^{\prime}$} \\
\hline $350 \mathrm{~m}$ & $110 \mathrm{ohms}$ \\
900 & 32 \\
3000 & 10 \\
\hline
\end{tabular}

TABLE VII

Received Energy and Audibility of Signals in Magnetic Detector

\begin{tabular}{c|c}
\hline$\underset{\mathrm{D}}{\text { Perikon }}$ & Magnetic \\
\hline $300 \mathrm{~mm}$ & 120 \\
90 & 53 \\
55 & 40 \\
20.5 & 16.5 \\
7.5 & 10 \\
2.0 & 2 \\
\hline
\end{tabular}


the couplings be made so loose that any further loosening will not decrease the value of the damping.

$\delta_{I}$ having been previously measured, the effective resistance $R^{\prime}$ of the circuit containing the magnetic detector was calculated from the formula

$$
\dot{R}^{\prime}=2 n L \delta_{I I}
$$

where $\delta_{I I}$ is the decrement, $n$ the frequency, and $L$ the inductance of the circuit. After subtracting the known ohmic high frequency resistance of the circuit, the other losses due to the detector for the different frequencies are shown in Table VI. This table would indicate that the amount of iron and the number of turns of the oscillatory circuit of the magnetic detector should be varied according to the frequency for which it is to be used.

In Table VII is shown the relation between loudness of signal as determined by the shunted telephone method in the magnetic and the oscillatory energy passing through it as determined by the deflections of the perikon galvanometer. It is seen that the loudness of signal in the telephone attached to the magnetic is not proportional to the square of the oscillatory current, but corresponds more nearly to the I.4 power.

THE FLEMING VACUUM VALVE DETECTOR. (Fig. 5)

The vacuum valve detector furnished for the test was constructed with fixed coupling coils inclosed in its case, so that it was found impossible to test it fairly at any except very short wave lengths without taking it completely to pieces.

The circuits were arranged as shown in Fig. 2, the vacuum valve being coupled by its own coils to circuit $I I$. Signals were taken alternately on the perikon, adjusted for maximum strength of signal, and on the vacuum detector, one detector circuit being removed when the other was in action. At a wave length of 350 meters and with a decrement of the incoming signals of 0.16 , the ratio of sensitiveness of the perikon to that of the vacuum detector, as determined by the variable coupling method, was almost exactly two to one. For a wave length of 900 meters the perikon appeared to be at least ten times as sensitive as the vacuum 
valve. As there is no reason to believe that the vacuum detector would change its sensitiveness with frequency, this difference is almost certainly due to insufficient coupling. ${ }^{8}$

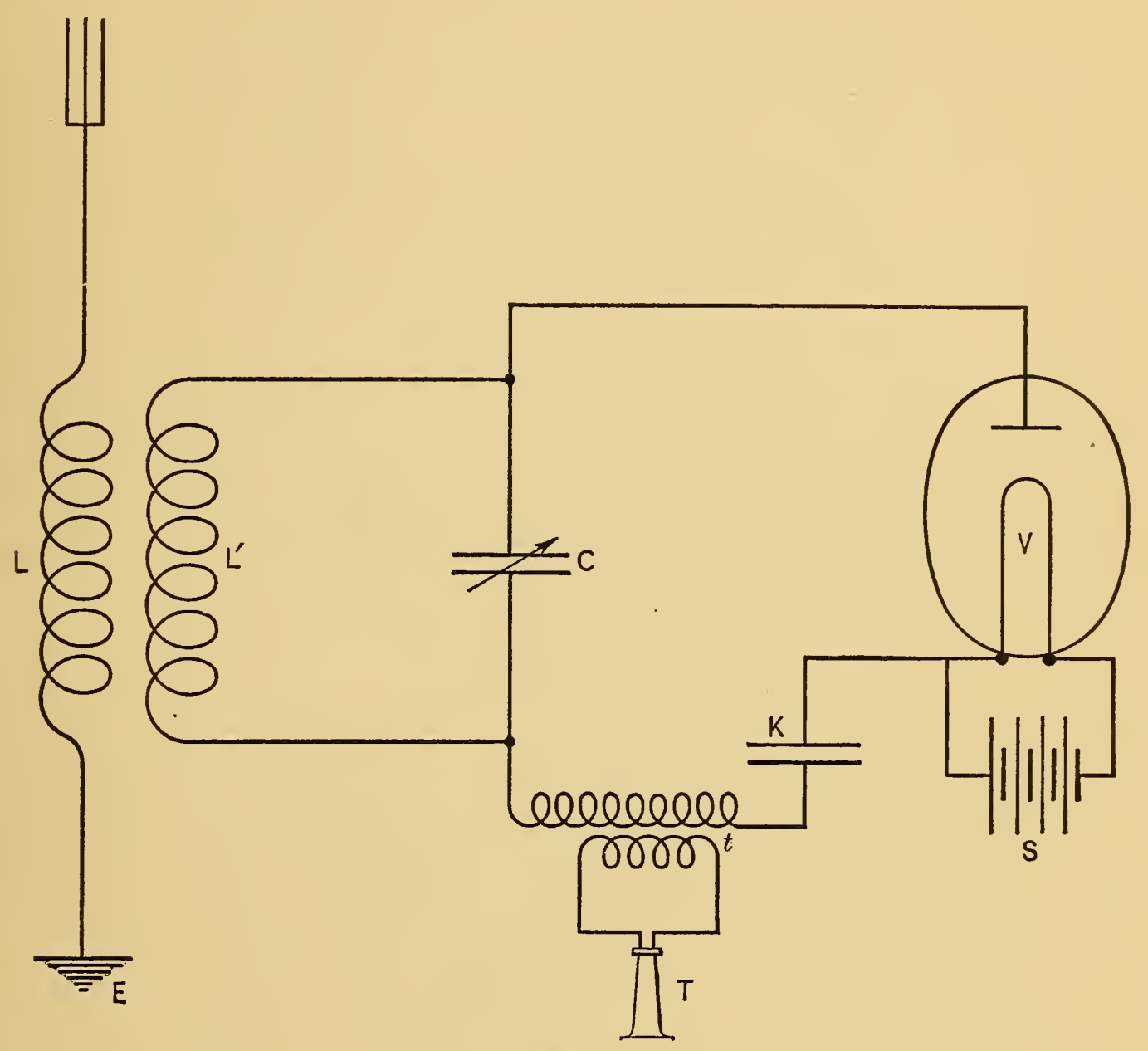

Fig. 5

In Table VIII is given the relation between oscillatory energy in the main circuit and the audibility of signals in the telephone of the vacuum detector, as determined by the shunted telephone method. The audibility is seen to be proportional to the energy. The telephones furnished with the vacuum detector had a resistance of $8300 \mathrm{ohms}$ and were connected by means of a step-up transformer to the detector circuit.

\footnotetext{
${ }^{8}$ This shows the disadvantage of constructing receiving apparatus with fixed coupling.$$
48848^{\circ}-10-18
$$ 
TABLE VIII

Received Energy and Audibility of Signals in Fleming Vacuum Detector

\begin{tabular}{c|c}
\hline $\begin{array}{c}\text { Perikon } \\
\text { D }\end{array}$ & Vacuum detector \\
\hline 43 & 38 \\
21 & 20 \\
10 & 9 \\
5 & 5 \\
\hline
\end{tabular}

THE AUDION. (Fig. 6)

The De Forest form of vacuum detector, called by him the audion, is shown diagrammatically in Fig. 6. Here $V$ is an exhausted glass bulb containing a tantalum filament $F$, heated by three storage cells $S$, a metal plate $P$ and a metal grid $G$ be-

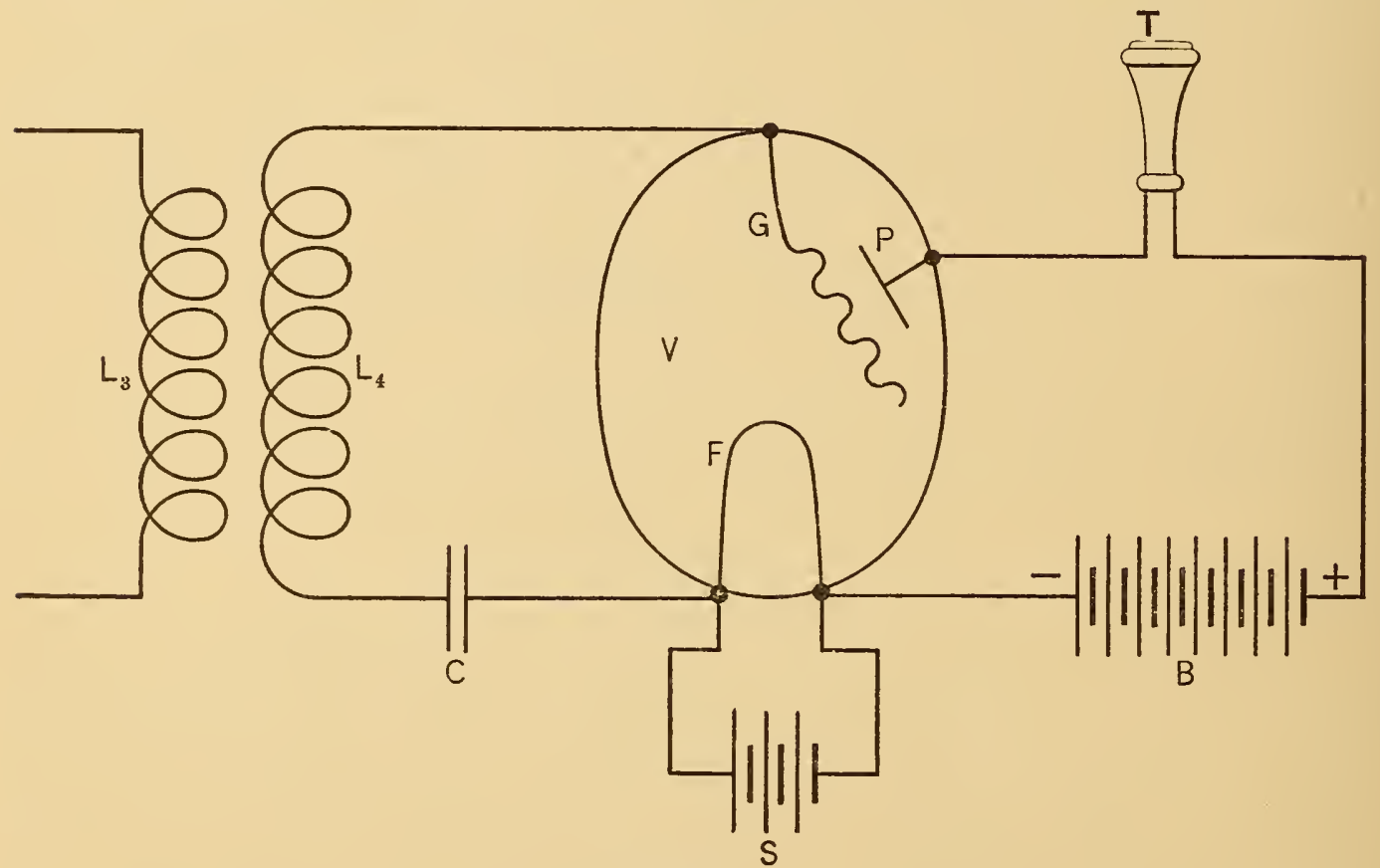

Fig. 6

tween the filament and the plate. The filament and plate are connected externally through a battery $B$ of 36 small cells, any number of which can be thrown into the circuit, and a pair of head telephones of about $\mathrm{I} 200 \mathrm{ohms}$ resistance. The filament 
and grid are connected through a small fixed condenser $C$ and an inductance $L$, which is coupled to the oscillatory circuit.

The action of the detector appears to be as follows: The heated filament $F$ is charged negatively by the battery $B$ and a steady stream of negative electrons flows from the hot filament to the cold plate producing a direct current through the telephone. When an oscillatory electromotive force is impressed on the circuit $F L_{4} G$, the negative current can flow from $F$ to $G$ inside the bulb and not in the reverse direction, just as in the Fleming detector. The grid $G$ thus becomes negatively charged and as it lies between $F$ and $P$ the direct current through the telephone is decreased as may be shown by placing a galvanometer in the telephone circuit.

The sensitiveness of the audion was compared with that of the electrolytic already described by the varying coupling method. (See Fig. 2.) Here $L_{3}$ and $L_{4}$ were the primary and secondary of a Wireless Specialty Apparatus Company tuner, the secondary condenser of the tuner being detached. The detectors were introduced alternately in circuit III and their most favorable conditions of coupling were determined separately. The audion, having a higher resistance, required a stronger coupling. The same telephones (r 200 ohms) were used for both detectors, previous tests having shown that they were suited to each. Highresistance telephones up to 8000 ohms were tried with the audion, but were found to be less satisfactory than the ones used. This is remarkable, as with the Fleming vacuum detector $8000-0 h m$ telephones are used and even then the current is stepped down through a transformer.

Below are given the data of the experiment:

$\lambda=900 \mathrm{~m} ., \delta_{1}=0.3, L_{1}=0.062, L_{2}=0.03, L_{3}=0.705, L_{4}=0.92 \mathrm{~m} . \mathrm{h}$. (electrolytic). $L_{4}=2.3$ (audion).

Electrolytic coupling $L_{3} L_{4}=7 \mathrm{~cm}$ on coupling scale ${ }^{9}$ of tuner; audion coupling $=2 \mathrm{~cm}$. Distance between coils $L_{1} L_{2}$ for least audible signal, $20.5 \mathrm{~cm}$ (electrolytic); $23 \mathrm{~cm}$ (audion).

\footnotetext{
${ }^{9}$ The numbers on the scale represent the distance the two coils are drawn apart from the position of maximum mutual inductance.
} 
Ratio of energy sensitiveness $\frac{\text { audion }}{\text { electrolytic }}=1.5$. Some of the bulbs were slightly more sensitive than this when first tried, but the sensibility decreased with use.

The law governing the relations between the intensity of the oscillations and the loudness of response in the telephone, as determined by the shunted telephone method, seems to depend on the impressed direct electromotive force from the battery $B$ and also on the temperature of the filament $F$, as would be expected from the known form of the current voltage curve in the case of the passage of electricity from a hot to a cold body through a gas. For most adjustments the response of the telephone is apparently nearly proportional to the square of the oscillatory current.

In addition to the above detectors, a very large number of other types have been tested in the laboratory, among which are the tellurium, silicon, and carbon already described, ${ }^{10}$ and also many crystal detectors. These detectors are all of the rectifying type and are remarkable in that when properly adjusted and used with telephones of proper resistance, and in some cases with small EMF in the direction of the rectification, they all give practically the same sensibility as the perikon. The usefulness of several of these is, however, limited by the difficulty of adjustment and a lack of constancy. In most of these detectors the loudness of the signal increases nearly in proportion to the square of the oscillating current, although in some of them the increase in loudness is less rapid.

United States Naval, Wireless Telegraphic Laboratory, March I, I9.10. 